\title{
Improved Multiscale Entropy Technique with Nearest-Neighbor Moving-Average Kernel for Nonlinear and Nonstationary Short-Time Biomedical Signal Analysis
}

\author{
S. P. Arunachalam $\mathbb{D}^{1},{ }^{1}$ S. $\mathrm{Kapa}^{2}{ }^{2}$ S. K. Mulpuru, ${ }^{3}$ P. A. Friedman, ${ }^{2}$ and E. G. Tolkacheva $\mathbb{D}^{1}$ \\ ${ }^{1}$ Department of Biomedical Engineering, University of Minnesota, Minneapolis, MN, USA \\ ${ }^{2}$ Department of Cardiovascular Medicine, Mayo Clinic, Rochester, MN, USA \\ ${ }^{3}$ Department of Cardiovascular Medicine, Mayo Clinic, Phoenix, AZ, USA
}

Correspondence should be addressed to S. P. Arunachalam; poigaiarunachalam.shivaram@mayo.edu

Received 7 April 2017; Revised 8 December 2017; Accepted 21 December 2017; Published 5 March 2018

Academic Editor: Arindam Bit

Copyright ( $\odot 2018$ S. P. Arunachalam et al. This is an open access article distributed under the Creative Commons Attribution License, which permits unrestricted use, distribution, and reproduction in any medium, provided the original work is properly cited.

\begin{abstract}
Analysis of biomedical signals can yield invaluable information for prognosis, diagnosis, therapy evaluation, risk assessment, and disease prevention which is often recorded as short time series data that challenges existing complexity classification algorithms such as Shannon entropy (SE) and other techniques. The purpose of this study was to improve previously developed multiscale entropy (MSE) technique by incorporating nearest-neighbor moving-average kernel, which can be used for analysis of nonlinear and non-stationary short time series physiological data. The approach was tested for robustness with respect to noise analysis using simulated sinusoidal and ECG waveforms. Feasibility of MSE to discriminate between normal sinus rhythm (NSR) and atrial fibrillation (AF) was tested on a single-lead ECG. In addition, the MSE algorithm was applied to identify pivot points of rotors that were induced in ex vivo isolated rabbit hearts. The improved MSE technique robustly estimated the complexity of the signal compared to that of SE with various noises, discriminated NSR and AF on single-lead ECG, and precisely identified the pivot points of ex vivo rotors by providing better contrast between the rotor core and the peripheral region. The improved MSE technique can provide efficient complexity analysis of variety of nonlinear and nonstationary short-time biomedical signals.
\end{abstract}

\section{Introduction}

Biomedical signals are characteristic of their corresponding physiological events and carry specific signatures [1]. Consequently, deciphering signal characteristics provides information regarding underlying processes that can be useful to inform or guide therapy. Most physiological processes are characterized by specific signals that reflect the nature and activities of such processes, which can contain biochemical, electrical, or physical information coming from molecular, cellular, organ, or systemic level sources [2]. Hence in a disease state, alterations to these physiological processes yield signal signatures that are different in some aspects from the normal state [1]. Electrocardiogram (ECG), electroencephalogram (EEG), electromyogram (EMG), electroretinogram, and so on are some examples of electrical signals that are commonly acquired for risk assessment, prognosis, diagnosis, therapy evaluation, and prevention of various diseases [3].

Biomedical signal analysis requires accurate quantification of the system state to distinguish between normal and pathological function or to predict the future state of the system using only short time series data that may only last a few seconds. Signal analysis is typically complicated by contamination with electromagnetic interference, power line interference, zero mean white noise, pink noise, brown noise from electrode movement, and other random noise [2]. 
Many biomedical signals are captured only for 3-8s and therefore are short nonstationary and/or nonlinear time series data, which prevent ordinary biomedical analysis algorithms from completely capturing their intrinsic complexity. For instance, Shannon entropy (SE) is commonly used for biomedical complexity analysis of EEG and ECG recordings [3-5]. However, one of the major limitations of the SE approach is related to the specific characteristics of the nonstationary and/or nonlinear time series data that work well for long but is not robust for short data segments. Several other symbolic dynamic approaches that use various entropy-based measures, such as Kolmogorov entropy, spectral entropy, wavelet entropy, permutation entropy, approximate entropy, and sample entropy, have been proposed to capture the intrinsic dynamics of nonstationary time series data to quantify their complexity [6-12]. However, it has been shown that these various entropy-based methods are efficient only for long time series and do not completely capture the complexities of shorter nonstationary time series data [13].

Recently, a multiscale entropy (MSE) technique was proposed for coarse-grained time-scaling procedures to offer more robust determination of the complexity of time series data [14]. Such coarse-graining procedures may result in invalid entropy value estimation for shorter time series; and this limitation was addressed by implementing a moving-average time series estimate [15]. However, the moving average in prior work was only performed in the forward direction, which can lead to significant underestimation of the complexity information that is present in the time series data [15]. Several variants of MSE have thus been proposed [16], but all of them provide only slight modifications from the original technique [15] and specifically depend on a one-sided moving average, which yields biased entropy estimates over different time scales. Several variants of MSE have been applied to test synthetic biomedical datasets without a rigorous demonstration of their feasibility for a biomedical application [17-19]. Therefore, using entropy-based techniques for rigorous complexity analysis of a biomedical signal in normal and diseased states has been very limited. Several researchers have used MSE technique for a variety of analysis using cardiac signal analysis [20-25] showing some promise for complexity assessment to aid diagnosis. However, the authors identify a major limitation of these MSE variants with the systematic bias in the one-sided average which may have affected the results. The introduced bias becomes extremely important to consider for improvement because most biological signals embed only subtle changes in short time series data which may have significant diagnostic potential that could be lost with such bias.

The challenge with short time series data analysis comes from the fact that the complexity of the data may not embed in the raw signal. Previously developed MSE techniques were introduced with time-averaged time series over multiple time scales for short time series analysis [15]. However, forward averaging introduces a systematic bias in the complexity estimation. To overcome this limitation, we proposed a nearest-neighbor moving-average kernel to better capture the complexity of nonlinear, nonstationary short time series data. We introduce the concept of "memory" by taking into account the past and future time series value while computing the nearest-neighbor moving average for time series data. Therefore, we introduce the time-scale factor " $\tau$ ", which represents time scaling in both forward and reverse directions with respect to a particular time point. Once this new time series is derived, the MSE estimate can be obtained by calculating the entropy of the new time series sample over multiple time scales to fully capture the intrinsic complexity of nonlinear and nonstationary time series data.

In this work, we propose an improved MSE technique, which includes significant and robust modification of the previously described MSE techniques. Specifically, we propose computation of the new time series with a nearestneighbor moving-average kernel that uses information from the "past" and "future" values to accurately capture the intrinsic dynamics of the short time series. Our modification will allow a robust analysis of nonlinear and nonstationary time series.

The efficacy and robustness of the improved MSE technique will be validated by performing noise analysis with respect to white, pink, and brown noise, which are commonly present in cardiac signals such as the ECG. Since SE has been used widely for biomedical signal complexity analysis so far, we will use it as a "gold standard", and we will compare the performance of the novel MSE technique with SE. We further hypothesized that the improved MSE technique will robustly quantify the complexity of nonlinear and nonstationary short time series data. We tested this hypothesis by applying the improved MSE technique for the analysis of the two physiological applications: (i) discrimination between normal sinus rhythm (NSR) and atrial fibrillation (AF) using a single-lead ECG and (ii) the accurate identification of the pivot point of rotors, which are potential ablation targets for AF and other arrhythmias.

\section{An Improved MSE Technique with Nearest-Neighbor Moving-Average Kernel}

The improved MSE algorithm consists of several steps as described below. Let $x=\left\{x_{1}, x_{2}, x_{3}, \ldots, x_{N}\right\}$ represent the electrogram time series of length $N$.

(1) Nearest-neighbor moving-averaged time series $z^{\tau}$ is computed for the chosen time-scale factor " $\tau$ " as illustrated in Figure 1 using the following equation:

$$
z_{j}^{\tau}=\frac{1}{(2 \tau+1)} \sum_{i=j}^{2 \tau+1} x_{i}
$$

where $1 \leq j \leq N-\tau$ and $i=1,2,3, \ldots, N$; Figure 1 shows the schematic to obtain the nearest-neighbor moving-window-averaging approach to obtain the new time series. 


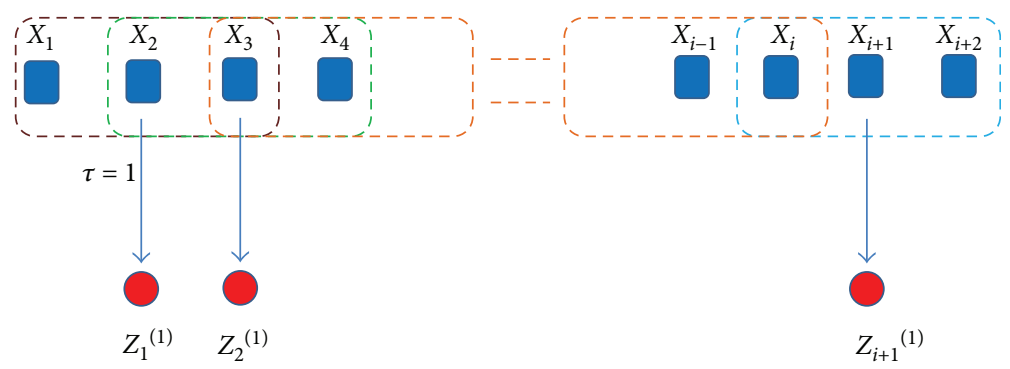

FIGURE 1: Schematic illustration to produce nearest neighbor moving-average time series with scale factor $\tau=1$ for the MSE algorithm. Blue squares represent raw time series data, and red dots represent the nearest-neighbor moving-averaged time series from which MSE is obtained. Brown squares represent the moving-window-averaging kernel for the raw second time point $\left(X_{2}\right)$ that averages one neighbor on both sides with $\tau=1$ to produce the first new time series point $Z_{1}^{(1)}$. Similarly, green square produces $Z_{2}^{(1)}$ and so on (orange square) with the blue square producing the last time series point $Z_{i+1}{ }^{(1)}$.

(2) Template vectors $\mathbf{y}_{k}^{m}(\delta)$ with dimension $m$ and delay $\delta$ are constructed from $\mathrm{z}^{\tau}$ (see Figure 1) at each specific $\tau$ as the following:

$$
\mathbf{y}_{k}^{m}(\delta)=\left\{z_{k} z_{k}+\delta \cdots z_{k}+(m-1) \delta\right\}
$$

where $1 \leq k \leq N-m \delta$.

(3) The Euclidean distance $d_{i j}^{m}$ for each pair of template vectors $\left\{\mathbf{y}_{i}^{m}, \mathbf{y}_{j}^{m}\right\}$ is calculated using the infinity norm as below:

$$
d_{i j}^{m}(\delta)=\left\|\mathbf{y}_{i}^{m}(\delta)-\mathbf{y}_{j}^{m}(\delta)\right\|_{\infty},
$$

where $1 \leq i, j \leq N-m \delta$ and $j>i+\delta$.

(4) Matched template vector pairs $\left\{\mathbf{y}_{i}^{m}, \mathbf{y}_{j}^{m}\right\}$ are computed based on a predefined tolerance threshold $r$ as

$$
d_{i j}^{m}(\delta) \leq r
$$

In this manuscript, the value for $r$ is chosen to be 0.2 times the standard deviation of the raw time series $x$. The delay factor $\delta$ is chosen to be 1 . The total number of matched template vectors is computed and denoted by $n(m, \delta, r)$.

Steps 2-4 are then repeated for $m+1$ dimension, and the total number of matched template vectors being computed is denoted by $n(m+1, \delta, r)$.

Finally, the improved MSE is calculated as the following:

$$
\operatorname{MSE}(x, m, \delta, r)=-\ln \frac{n(m+1, \delta, r)}{n(m, \delta, r)} .
$$

\section{Materials and Methods}

3.1. Noise Analysis. We evaluated the performance of the improved MSE technique and compared it with the performance of SE approach with respect to the most common sources of noise: (i) zero mean white noise, (ii) pink noise which has the inverse frequency response ( $1 / f)$, and (iii) brown noise which has the inverse frequency squared response $\left(1 / f^{2}\right)[26,27]$.
White, pink, and brown noises were simulated in MATLAB $^{\mathrm{TM}}$, with 10,000 sample points. Ten short time series (TS) versions of these data were created with 250, 500, 750, $1000,2000,4000,5000,6000,8000$, and 10,000 samples. MSE was calculated via (5) for each noise using different time-scale factors " $\tau$ " from 1 to 20 over varying time series lengths. Normalized MSE (for $\tau=1,2,3,5$ ) and SE were calculated by dividing the MSE (and SE) values by the maximum value of MSE (and SE) across varying time series. MSE and SE results for $\tau>5$ are quantitatively similar to that of $\tau=5$ and therefore are not shown.

3.2. Description of Datasets for Noise Analysis. To test the robustness of an improved MSE technique in the presence of various noises, we used (1) simplified non-physiological sinusoidal wave and (2) physiological ECG signal, which is the most commonly used time series signal for the diagnostic of various diseases of the heart.

(1) A sinusoidal wave with single frequency of $10 \mathrm{~Hz}$ and a multifrequency sinusoidal wave with superposition of $2,5,10,15$, and $20 \mathrm{~Hz}$ frequencies were used. Ten short time series versions of the data were simulated in MATLAB.

(2) Noise-free flat baseline ECG was obtained using an electronic ECG simulator with 10,000 sample points at $250 \mathrm{~Hz}$ sampling rate. Ten short time series versions of these data were created.

White, pink, and brown noises were added to the noisefree signals and the analysis was performed as described in sub-Section A to compare the performance of MSE and SE techniques.

3.3. NSR and AF ECG Discrimination Analysis. Publically available ECG datasets were obtained from the MIT-BIH Physionet database during NSR and AF [28]. Ten NSR and AF datasets of 10 -second duration and $250 \mathrm{~Hz}$ sampling rate were used for analysis. The signals were not preprocessed for noise removal and $\tau=3$ for MSE calculation. NSR and AF datasets were compared using custom MATLAB software. Mann-Whitney test with $p$ value of 
0.01 was used for testing statistical significance and was performed using OriginPro software (OriginLab Corporation, Northampton, Massachusetts).

3.4. Optical Mapping Data from Isolated Rabbit Hearts. Optical mapping movies during a single rotor or figure-of8 reentry were obtained from an isolated rabbit heart by inducing ventricular tachycardia via burst pacing as described previously $[29,30]$. The movies were 3 -second long, acquired at 600 frames per second temporal and $64 \times 64$-pixel spatial resolution. Two-dimensional (2D) MSE maps were generated for both single rotor and figureof- 8 reentry using the MSE values with the scale factors $\tau=1,2$, and 3 at each pixel location across all the frames. For comparison purposes, the 2D SE map was computed. A custom MATLAB (MathWorks Inc., Natick, MA) program was developed for all processing. Supplemental videos SV1 and SV2 are provided for reference that shows the phase movie of single and double rotor, respectively.

\section{Results}

4.1. Noise Analysis. Figure 2 shows the robustness of MSE and SE techniques with respect to different types of noise: white (a), pink (b), and brown (c). The middle row of Figures 2(a)-2(c) shows the MSE values as a function of $\tau$ for varying TS lengths. As expected, for white noise, MSE monotonically decreases as $\tau$ increases, and changing TS length does not affect the data. For pink noise, MSE increases with the increase of the TS length, and for long TS (1000 samples), MSE does not depend on $\tau$. For brown noise, MSE decreases with the increase in the TS length and does not depend on $\tau$ for long TS. These results demonstrate the robustness of MSE since the expected behavior is observed for each noise. The bottom row of Figures 2(a)-2(c) shows the normalized values of MSE (for different $\tau$ ) and SE as a function of the TS length. As seen from these data, the values of SE decrease as TS decreases for all types of noises, while MSE values do not depend on the TS length. These results demonstrate that the performance of MSE is better than SE, especially for short time series.

The MSE of white noise is expected to show a monotonically decreasing response with higher scale factors [14-16] which was seen in Figure 2(a) middle panel with increasing scale factor due to the nearest neighbor averaging that leads to lower MSE for white noise is shown. For pink noise which has a $1 / f$ response, higher MSE than white noise is expected but with a constant value across multiple time scales [14]. As expected, MSE levels out at higher time series lengths above 1000 sample points across the different time scales seen in Figure 2(b) middle panel. This means that for a sampling rate of $250 \mathrm{~Hz}$, MSE can capture the complexity with just $4 \mathrm{~s}$ of data. Similarly, for brown noise, MSE is expected to be constant and as seen from Figure 2(c) middle panel after a TS length of 750 sample points, MSE is more or less the same across multiple time scales. Figures 2(a)-2(c) bottom panel demonstrates the fact that SE estimates lower values for short time series and gradually increases with increasing time series length for all three types of noise. MSE has higher values even for the shortest time series, thereby capturing the complexity better than SE. Overall, the results indicate that if at least 1000 sample points are available, MSE can capture the complexity robustly compared to SE. For most physiological monitoring, $250 \mathrm{~Hz}$ sampling frequency is common, which indicates that $4 \mathrm{~s}$ short time series data should be sufficient for robust analysis using MSE.

Figure 3 demonstrates the robustness of MSE compared with SE technique for single-frequency sinusoidal wave in the absence and presence of different noises. Figure 3(a) shows the single-frequency sinusoidal wave at $10 \mathrm{~Hz}$ for 500 sample points (left panel), the MSE values as a function of $\tau$ for varying TS (middle panel), and the normalized values of MSE (for different $\tau$ ) and SE as a function of TS length (right panel) in the absence of noise. These data demonstrate higher efficacy of the MSE technique in capturing the complexity of the sine wave than that of SE, which only works well for larger TS lengths. The robustness of the MSE and SE techniques in identifying the complexity of a single-frequency sinusoidal wave in the presence of noise is shown in Figure 3 for the white (b), pink (c), and brown (d) noises. The top row of Figures 3(b)-3(d) shows the amplitude of sinusoidal wave with noise, while the bottom row shows the normalized values of MSE (for different $\tau$ ) and SE as a function of TS length. Our results suggest that MSE captures the complexity of sinusoidal waves better than $\mathrm{SE}$ in the presence of these noises.

Figures 4 and 5 show the results for the multifrequency sinusoidal wave and the noise-free flat ECG, respectively. Similar to the response seen in Figure 2 for raw noise, (b)-(d) of Figures 4 and 5 demonstrate that SE is very small for short TS and gradually increases with increasing TS length, while MSE has high values even for the shortest TS, thereby capturing the complexity better than SE. The results demonstrate the efficacy of the novel MSE technique in quantifying the complexity of complex time series data in the presence of noise better than that of the commonly used SE approach.

4.2. ECG Analysis. Figure 6 shows the raw ECG with NSR (a) and $\mathrm{AF}(\mathrm{b})$. Note that visual inspection of these traces cannot be used to correctly discriminate between NSR and AF. Figure 6(c) shows the boxplot of MSE values for $10 \mathrm{AF}$ and NSR datasets demonstrating statistically significant differences $(p<0.01)$ and therefore accurate discrimination between NSR and AF. As observed in Figures 6(a) and 6(b) visually, it is difficult to interpret the difference between NSR and AF on the ECG as the chaotic nature of AF manifests itself into small morphological disturbances which need robust algorithms to effectively capture the complexity. MSE robustly discriminates NSR and AF.

4.3. Identification of Pivot Point of the Rotor. A snapshot of a phase movie of a single rotor in isolated rabbit heart is shown in Figure 7(a). In this movie, different colors represent different phases of the action potential, and the pivot point of the rotor can be easily identified as the point where different phases converge. Corresponding voltage traces from the core (pixel "1") and periphery of the rotor (pixel " 2 ") are also shown. At the core of the rotor, broader distribution of 

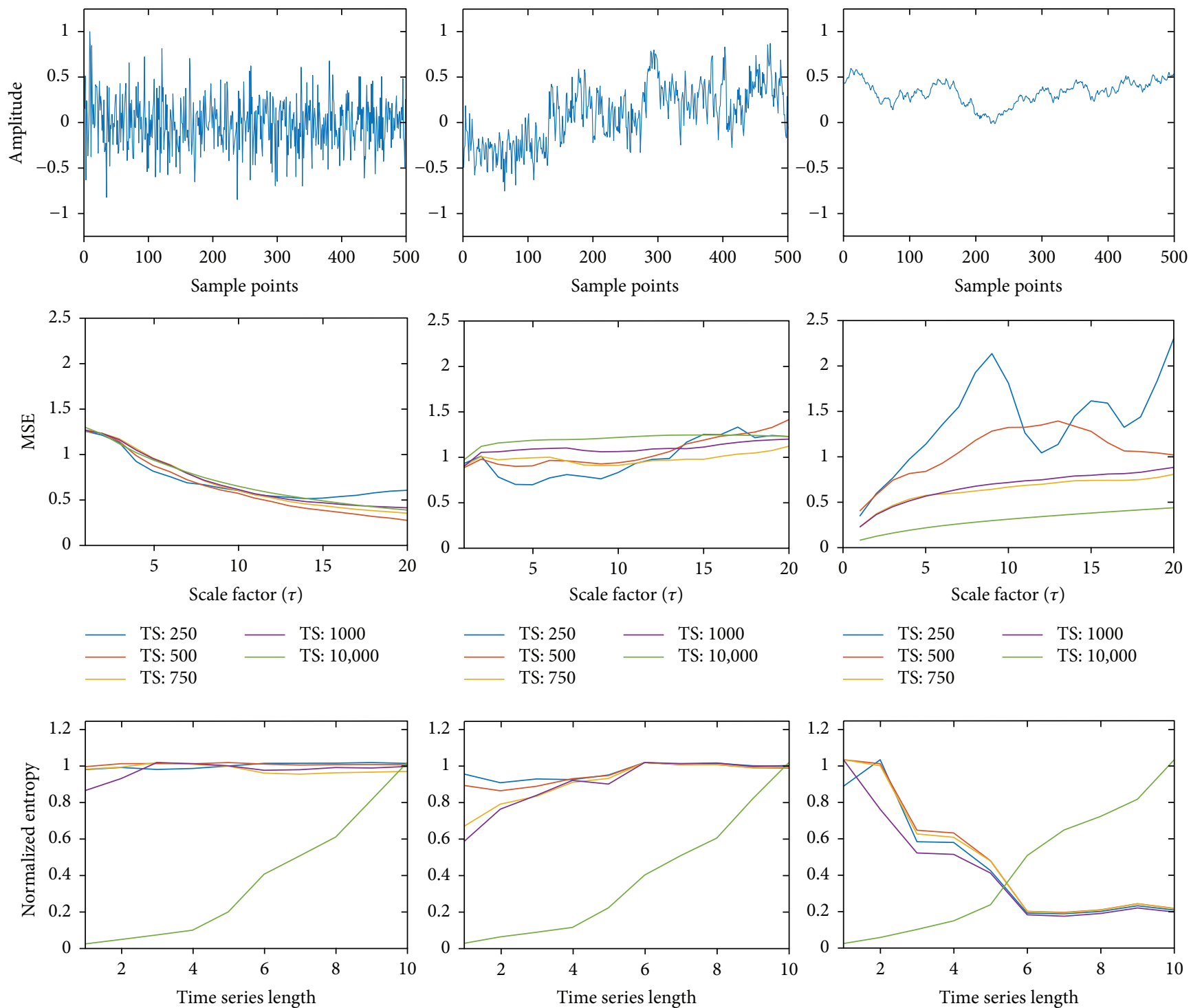

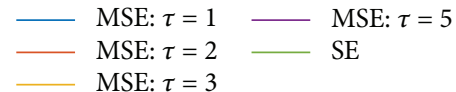

(a) White noise

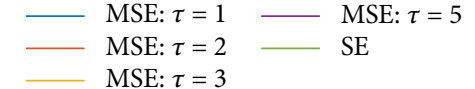

(b) Pink noise

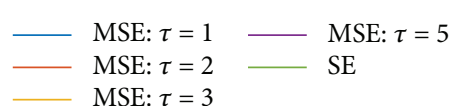

(c) Brown noise

FIgUre 2: (a) Top panel shows white noise with 500 sample points; Middle panel shows the MSE for various time series (TS) lengths; bottom panel shows normalized MSE (for scale factors $\tau=1,2,3$, and 5) and SE; (b) top panel shows pink noise with 500 sample points; middle panel shows the MSE for various time series (TS) lengths; bottom panel shows normalized MSE (for scale factors $\tau=1,2,3$, and 5) and SE; (c) top panel shows brown noise with 500 sample points; middle panel shows the MSE for various time series (TS) lengths; bottom panel shows normalized MSE (for scale factors $\tau=1,2,3$, and 5) and SE.

voltage amplitude occurs due to the chaotic nature at the rotor pivot point and therefore, a higher MSE value was expected. At the periphery of the rotor, more uniform electrical activity is observed and hence, a lower MSE value was expected. Figure 7(b) shows the 2D MSE maps for three time-scale factor $\tau=1,2$, and 3. Note the MSE technique can accurately identify the location of the pivot point of the rotor for each $\tau$. As seen from (b), the pivot point has higher MSE values than the periphery thereby enabling its precise localization, and higher values of " $\tau$ ” results in better contrast between the rotor core and periphery. Figure $7(\mathrm{c})$ shows the normalized 2D SE map of the same single rotor. It is important to note that although SE can correctly identify the pivot point of the rotor, the contrast between $\mathrm{SE}$ values at the core and the periphery is low, which challenges accurate identification.

Figure 8(a) shows a snapshot of a phase movie for an example of figure-of- 8 reentry in an isolated rabbit heart. Similar to Figure 7, one can see that the MSE technique can correctly identify the location of the pivot points of the rotors 

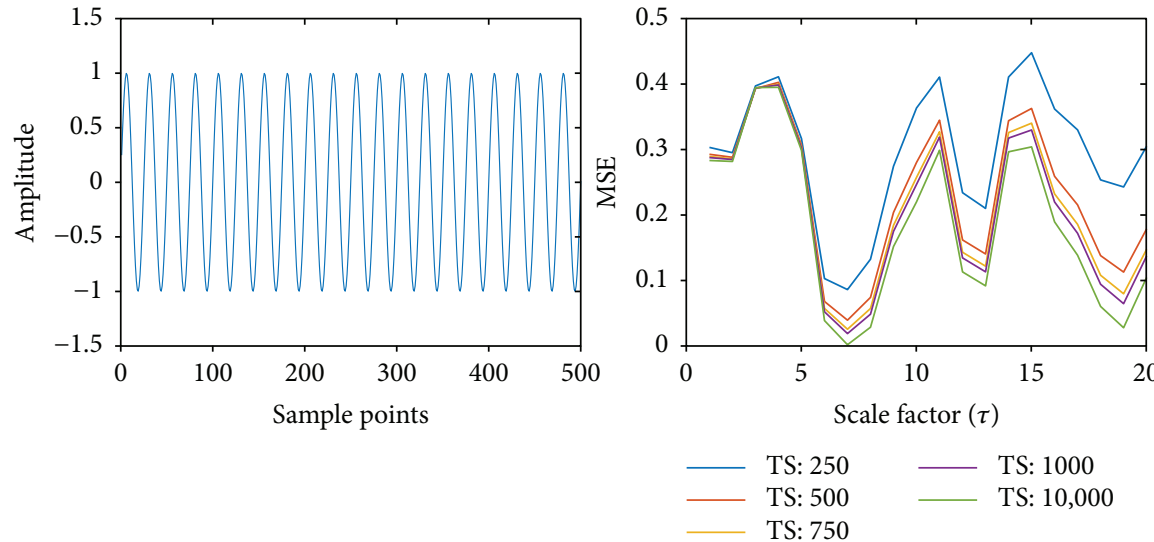

(a) Raw sinusoidal signal
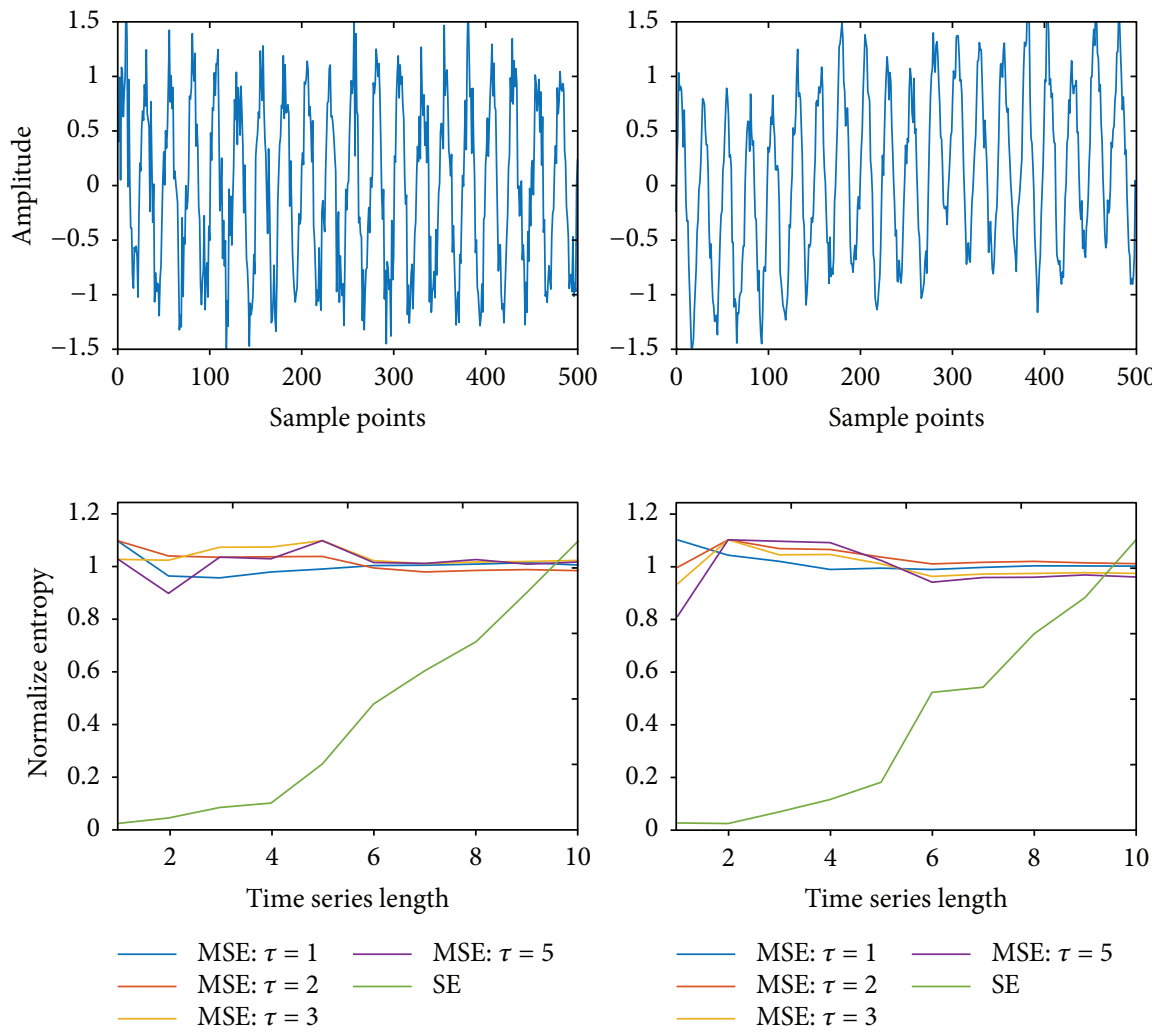

(b) White noise
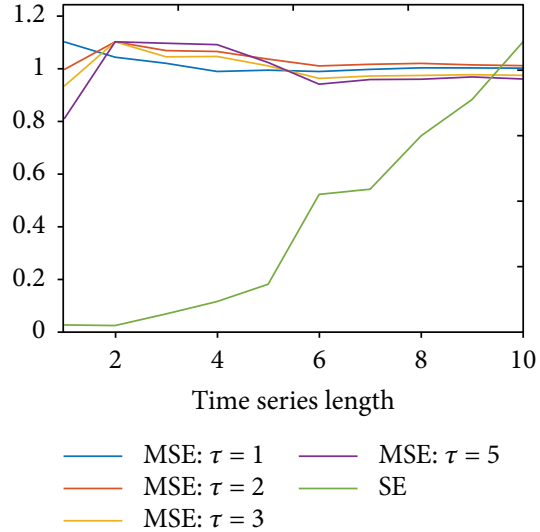

(c) Pink noise

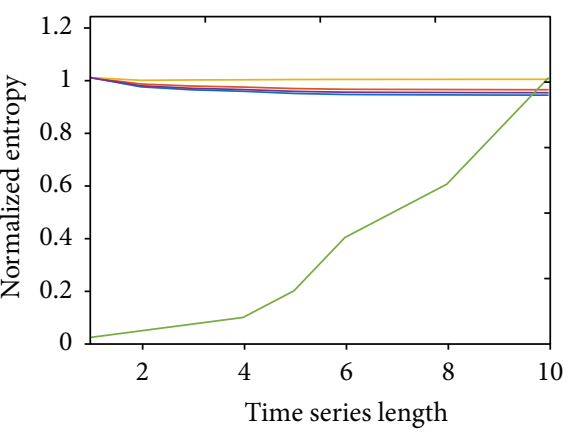

MSE: $\tau=1 \quad$ MSE: $\tau=5$

MSE: $\tau=2$

MSE: $\tau=3$
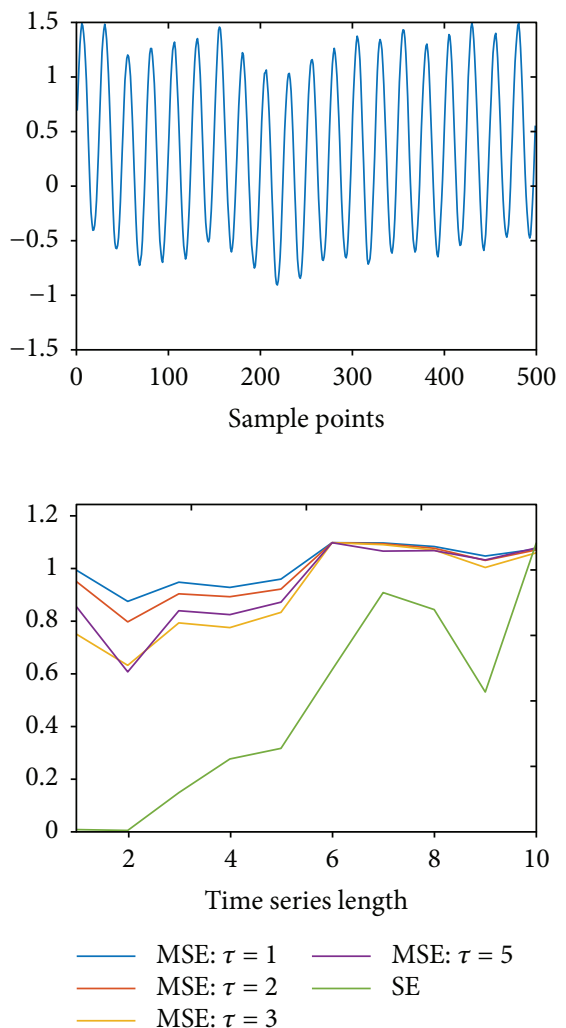

(d) Brown noise

FIGURE 3: (a) Left panel shows single-frequency $(10 \mathrm{~Hz})$ sinusoidal wave with 500 sample points; middle panel shows the MSE for various length time series across several scaling factors; right panel shows normalized MSE (for scale factors $\tau=1-10$ ) and SE; (b) top row shows sinusoidal wave with white noise, (c) pink noise, and (d) brown noise; (b) bottom row shows normalized MSE (for scale factors $\tau=1-10$ ) and SE for sine wave with white noise, (c) pink noise, and (d) brown noise, respectively.

for each $\tau$ and that the performance of the MSE technique is much better than SE observed in Figures 8(b)-8(c).

As seen in Figure 7(b), it is seen that a scale factor of $\tau=1$ was sufficient enough to provide the necessary contrast to identify the rotor pivot points with higher MSE values at the rotor pivot point than that in the periphery. Higher scale factor values provided improved contrast as seen when comparing 2D MSE maps in Figure 7(b). Similar results are observed for figure-of- 8 reentry data seen in Figure 8. It is interesting to note that at pixel location " 1 ," the rotor meanders to some extent which is also captured robustly by MSE compared to SE.

\section{Discussion}

In this study, we developed an improved MSE technique with nearest-neighbor moving-average kernel and demonstrated that it can be successfully used for the analysis of nonlinear 

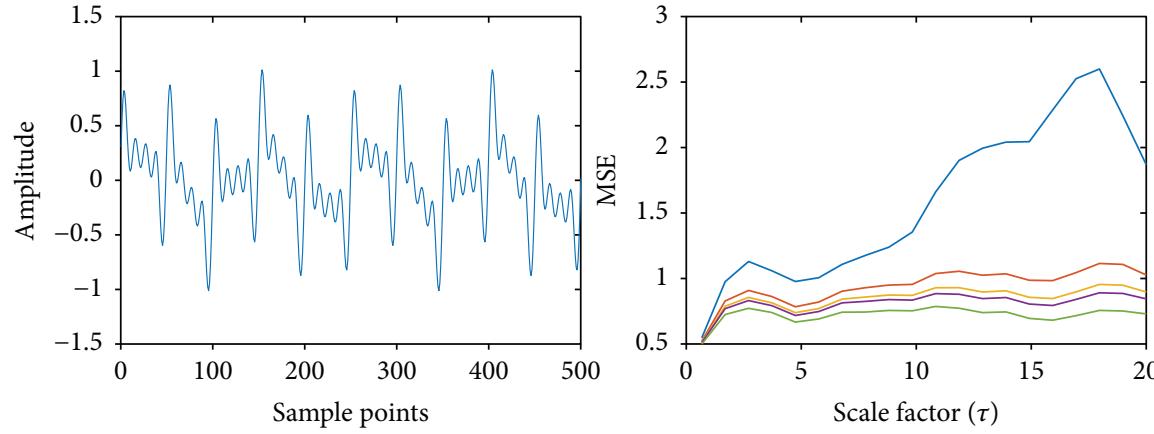

TS: 250
TS: 500

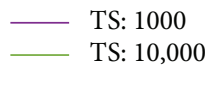

(a) Raw sinusoidal signal
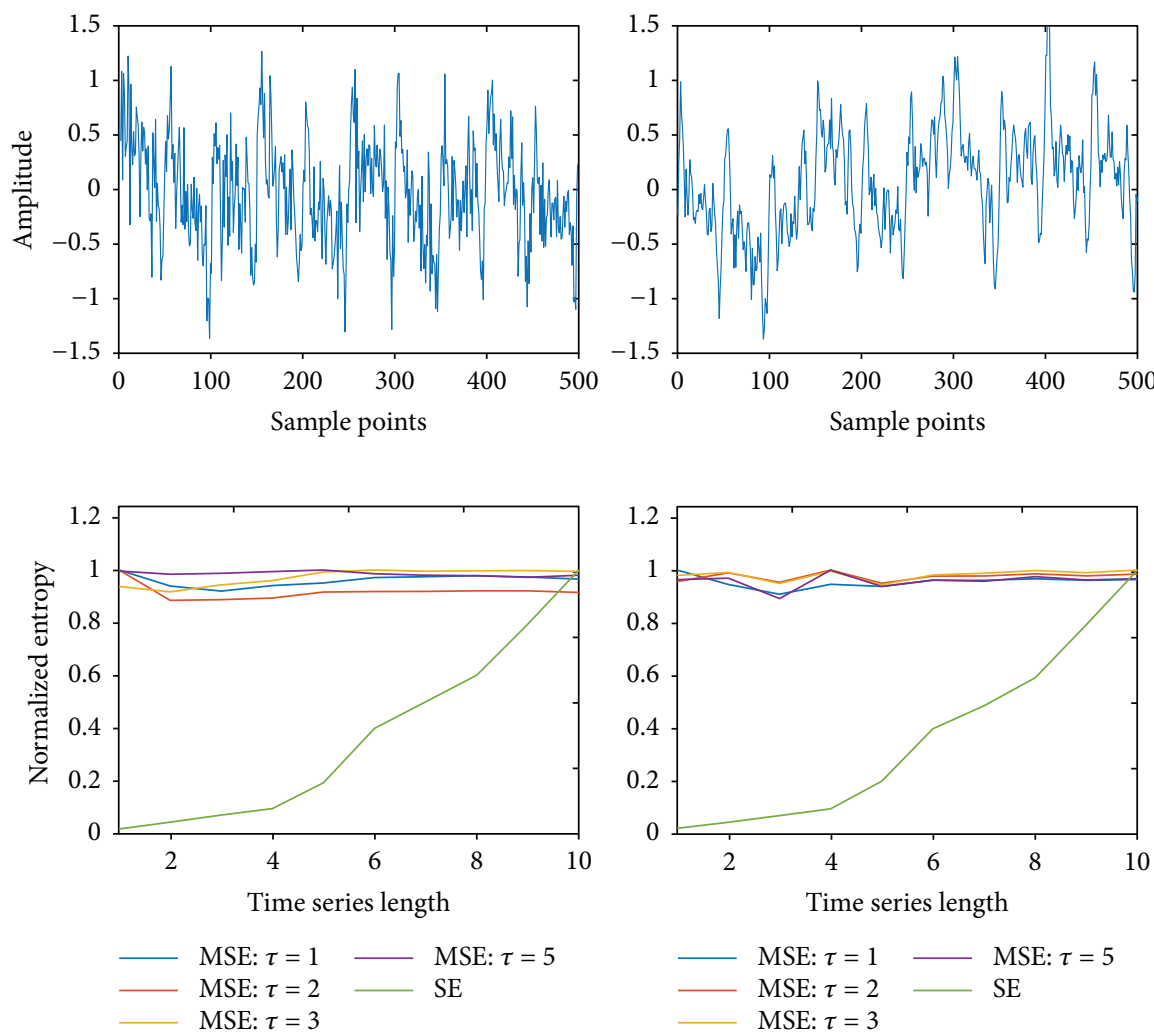

(b) White noise
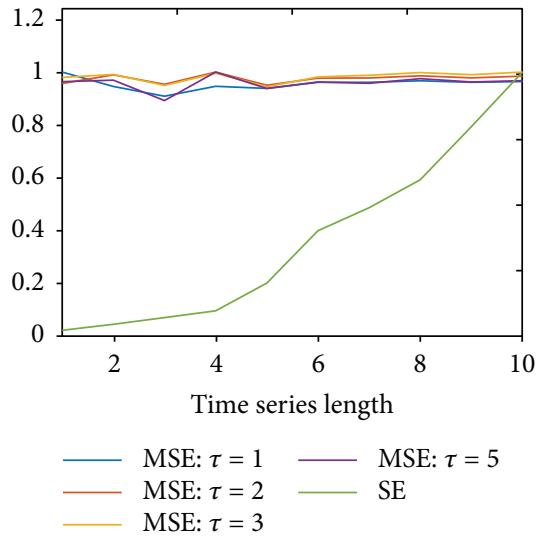

(c) Pink noise
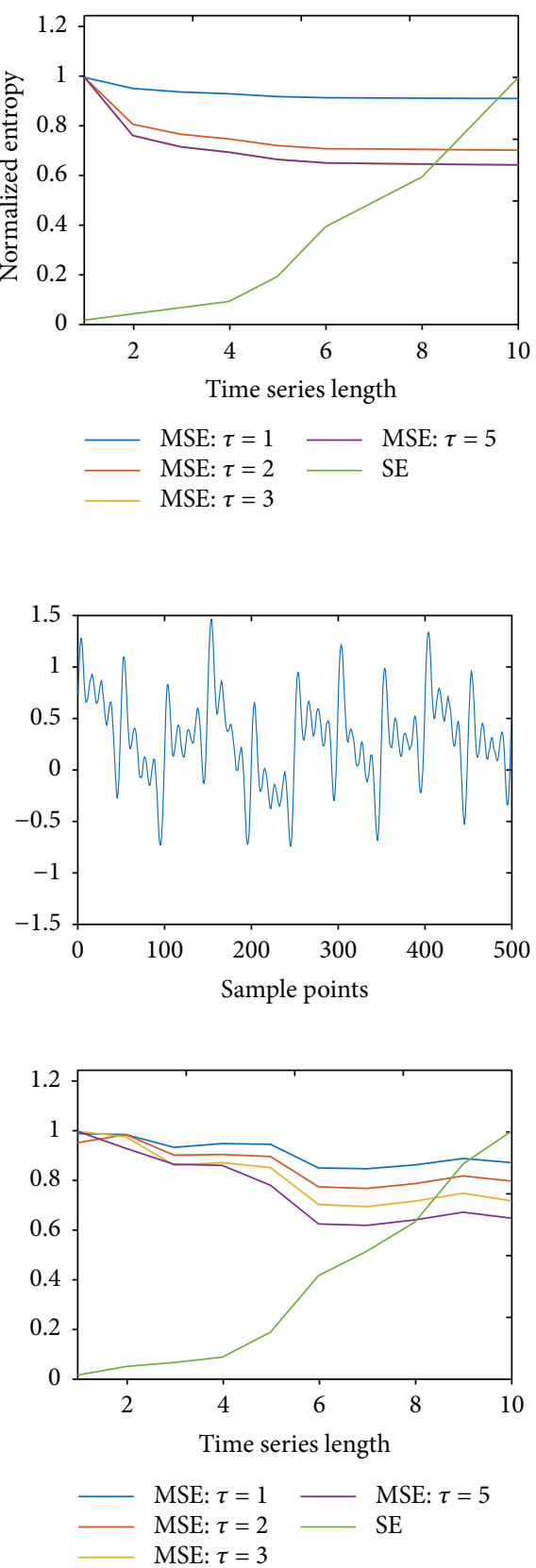

(d) Brown noise

FIGURE 4: (a) Left panel shows multifrequency sinusoidal wave with 500 sample points; middle panel shows the MSE for various length time series across several scaling factors; right panel shows normalized MSE (for scale factors $\tau=1-10$ ) and SE; (b) top row shows sinusoidal wave with white noise, (c) pink noise, and (d) brown noise; (b) bottom row shows normalized MSE (for scale factors $\tau=1-10$ ) and SE for sinusoidal wave with white noise, (c) pink noise, and (d) brown noise, respectively.

and nonstationary short time series physiological data. The MSE robustly estimated the complexity of short time series data compared to SE with various noises such as white, pink, and brown noises. Major findings of this manuscript are the following: (1) MSE discriminated NSR and AF on single-lead ECG of $10 \mathrm{~s}$ recordings without any preprocessing steps and (2) MSE precisely identified the pivot point of the rotor (single and figure-of- 8 reentry) with $3 \mathrm{~s}$ optical mapping data from isolated rabbit hearts by providing better contrast between the rotor core and the periphery region when compared to the SE approach. The efficacy of MSE technique was clearly demonstrated with short time series analysis which can be used in a variety of other physiological applications.

5.1. Sinusoidal Wave Analysis. Sinusoidal wave analysis is the most elegant approach to demonstrate the efficacy of the improved MSE technique over the conventionally used SE 

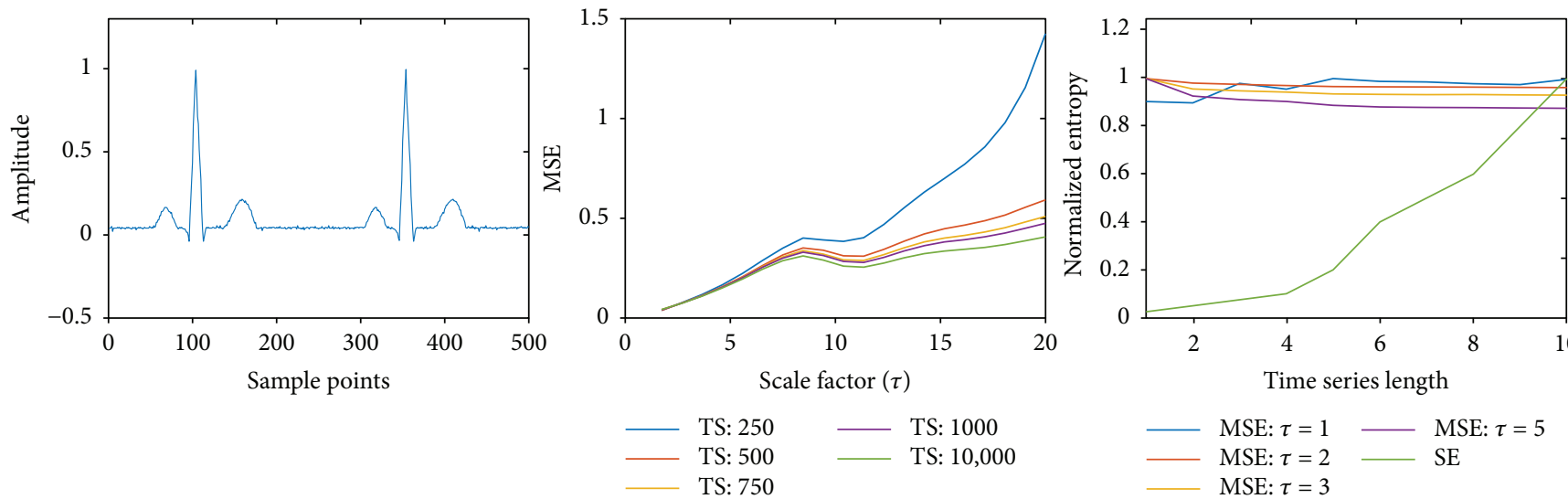

(a) Raw ECG signal
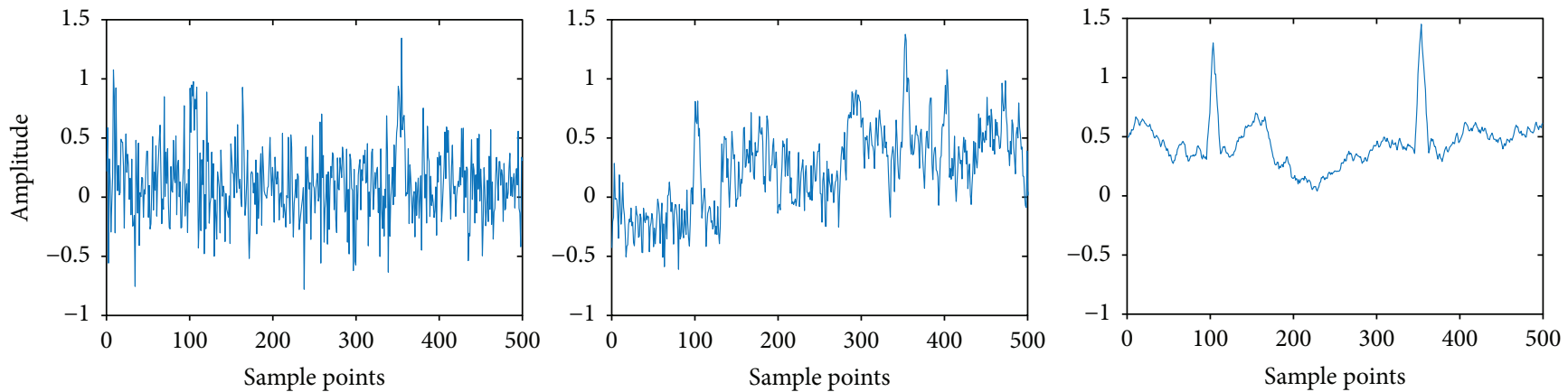

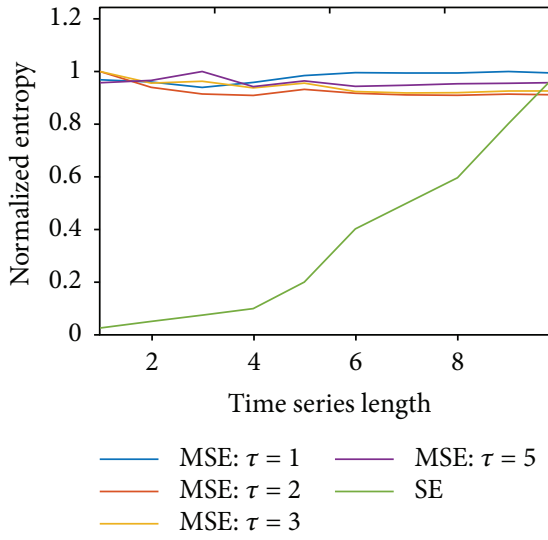

(b) White noise
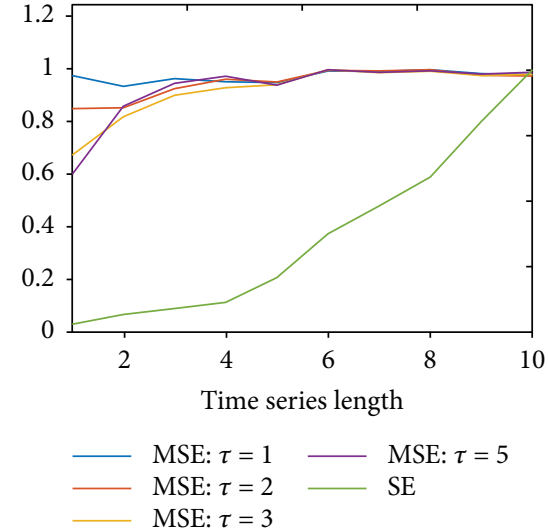

(c) Pink noise
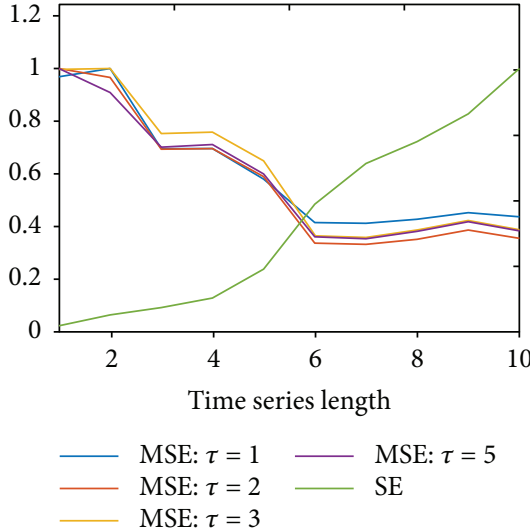

(d) Brown noise

FIGURE 5: (a) Left panel shows sample ECG trace with 500 sample points; middle panel shows the MSE for various length time series across several scaling factors; right panel shows normalized MSE (for scale factors $\tau=1-10$ ) and SE; (b) top row shows sinusoidal wave with white noise, (c) pink noise, and (d) brown noise; (b) bottom row shows normalized MSE (for scale factors $\tau=1-10$ ) and SE for sinusoidal wave with white noise, (c) pink noise, and (d) brown noise, respectively.

approach for short time series analysis of biomedical signals. We demonstrated that both for single-frequency and multifrequency sinusoidal waves with added noise, SE underestimated the complexity at short time series for all three noise cases and performed better at longer time series lengths. However, MSE was robust even at shorter time series with 1000 sample points in the presence of the three types of noise. The results suggest the value of MSE technique in analyzing complex short time series physiological signals that can be contaminated with these noises and its use for the prognosis and diagnosis of various disease states.

5.2. Noise-Free ECG Analysis. ECG analysis is very commonly used for a wide variety of cardiac conditions to yield information regarding the state of the heart. Since most remote and ambulatory real-time ECG monitoring present at most 3-5 seconds of ECG data, conventional complexity analysis methods such as SE are limited. However, we 


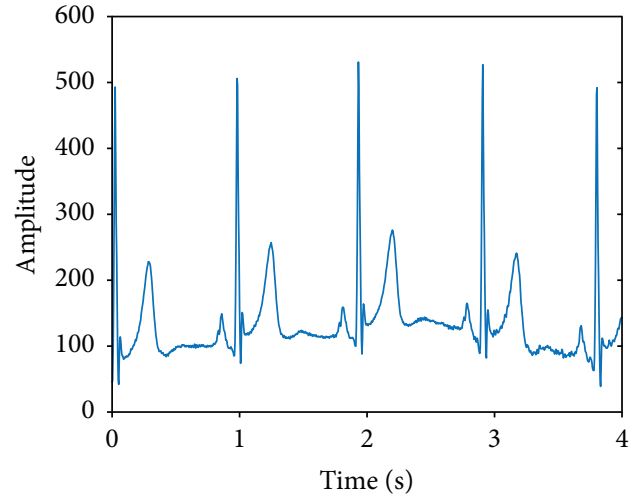

(a)

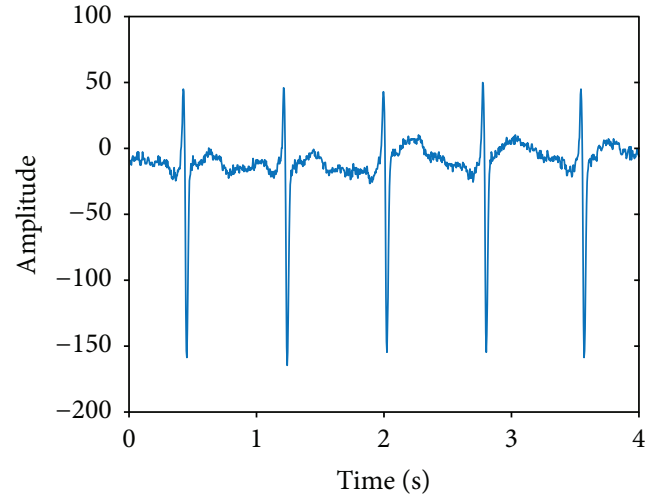

(b)

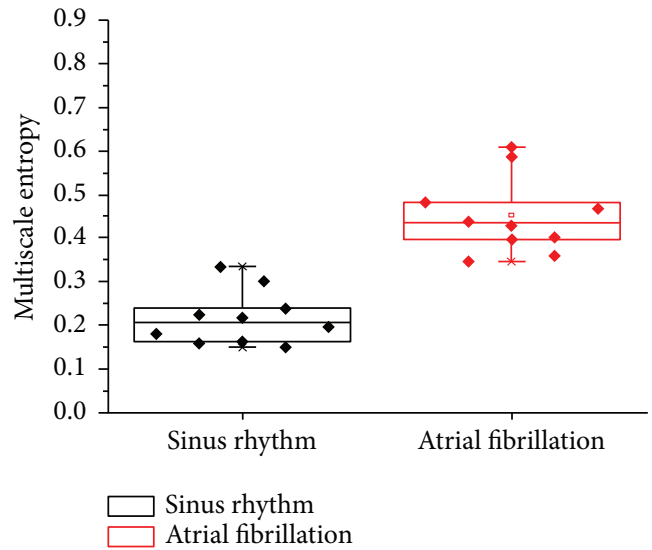

(c)

FIGURE 6: (a) Representative example of an ECG trace with normal sinus rhythm; (b) representative example of an ECG trace with AF; (c) box plot showing MSE values for sinus rhythm and AF ECG datasets. The NSR and AF were significantly different $(p<0.01)$ on MSE.

demonstrated that MSE robustly estimated the complexity of short time series ECG data even in the presence of noise.

5.3. Discrimination between NSR and AF. AF is the most common sustained cardiac arrhythmia that is associated with increased risk of stroke, heart failure, and death affecting more than 2.3 million people in the United States and over 30 million people worldwide [31]. Although the persistent form of AF can be detected relatively easy, detecting paroxysmal AF is often a challenge since continuous monitoring is required, which in turn requires methods to discriminate NSR from AF through large quantities of data [32].

Although there are several methods available for NSR and AF discrimination, they face limitations in successfully detecting AF with high sensitivity and specificity using short-time ECG data [32-34]. The major issues with these approaches are that they often distort the ECG by several preprocessing steps with filters, they do not provide reliable discrimination using short ECG time series data, and many of them lack real-time capability that makes it difficult to trust the data for diagnosis and treatment. Here, we demonstrated that the improved MSE technique can robustly discriminate AF from NSR using a single-lead ECG. The results motivate the application and use of this MSE technique for many hand-held and remote ECG monitors to autodetect AF.

5.4. Identification of Pivot Points of Rotors. Catheter ablation to treat paroxysmal AF has been shown to be up to $87 \%$ successful using pulmonary vein (PV) isolation [35-40]. However, in patients with persistent AF ablation, it is challenging since the location of the triggers is unclear, and it has been shown that triggers commonly arise outside the PVs. Recent research suggests that AF ablation has a success rate of $28 \%$ with $51 \%$ after multiple repeat procedures in persistent AF [41].

It is believed that rotors are caused by reentrant mechanisms which might be responsible for maintaining persistent AF. Identification of the rotor pivot point as a suitable ablation target has been the research focus for many investigators. However, these investigations are challenged with short time series data in the clinical setting. Here, we used optical mapping data in which rotors can be clearly visualized, and we demonstrated that the improved MSE technique can precisely identify pivot points in both single rotor and figure-of- 8 reentry, thus offering a robust mapping tool to guide identification of 


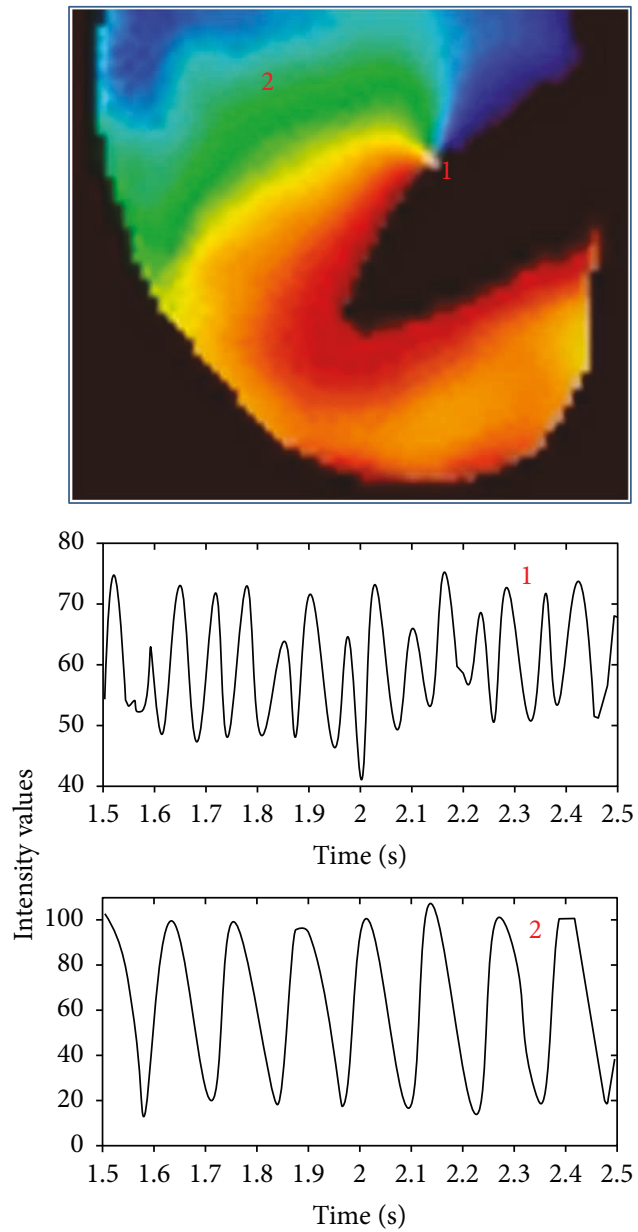

(a) Raw data from optical mapping
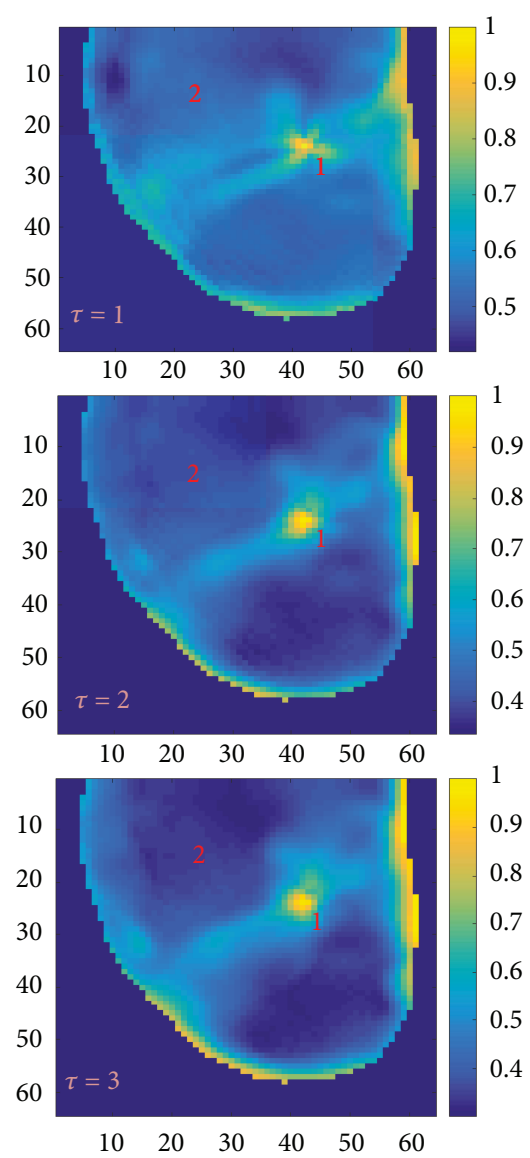

(b) 2D MSE maps

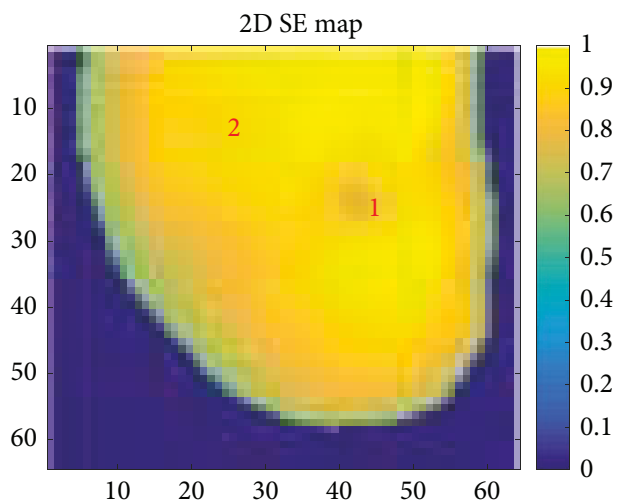

(c) 2D SE map

Figure 7: (a) Top panel shows a representative example of a single rotor. Pixel locations "1" represent rotor core region and "2" represent rotor periphery; bottom panel shows corresponding voltage traces at those pixel locations. (b) Normalized 2D MSE maps; top panel with scale factor $\tau=1$; middle panel with $\tau=2$; bottom panel with $\tau=3$, correctly identifying rotor core regions; (c) normalized 2D SE map with lower SE values at the rotor core.

AF ablation targets. In the clinical setting, electrogram recordings are frequently limited to 2.5-5-second segments due to the need for frequent catheter repositioning during the procedure, challenging conventional mapping approaches to precisely identifying substrates in $\mathrm{AF}$ and other arrhythmias.
5.5. Limitations. A limitation of the improved MSE technique is the need to select a correct choice of the time scale factor " $\tau$." Since the nearest-neighbor moving averaging is employed, large time scales will cause excessive smoothing of the data which may lead to loss of some complexity information. Therefore, caution should be used in the appropriate 

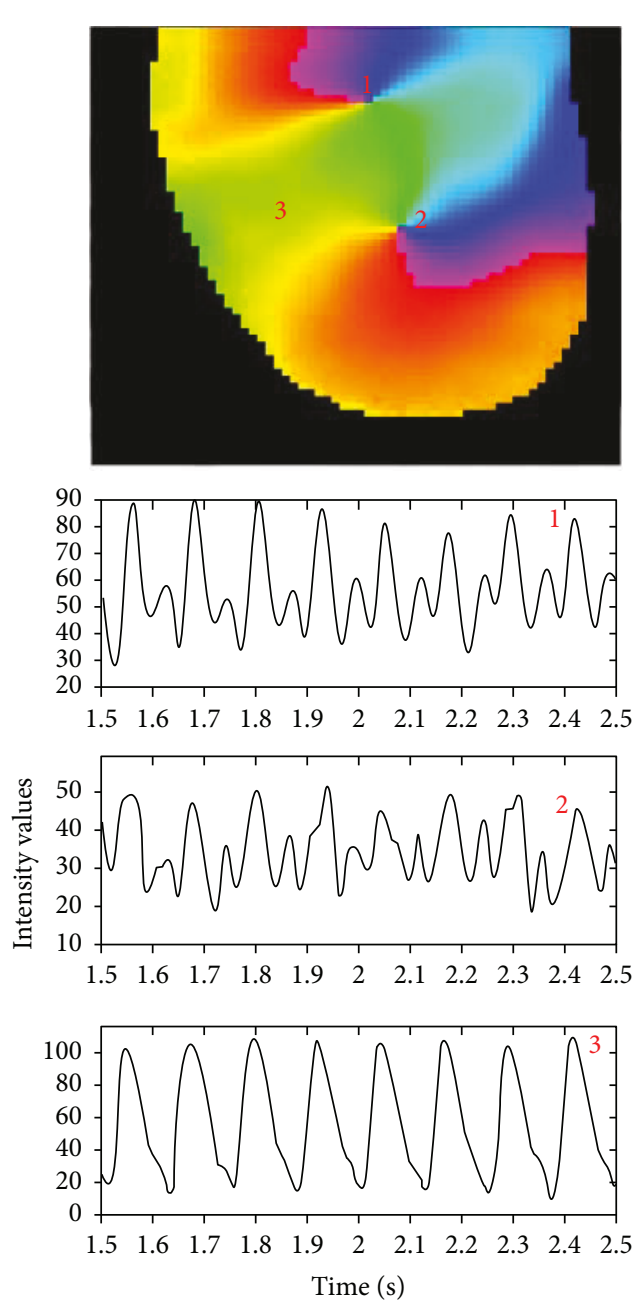

(a) Raw data from optical mapping

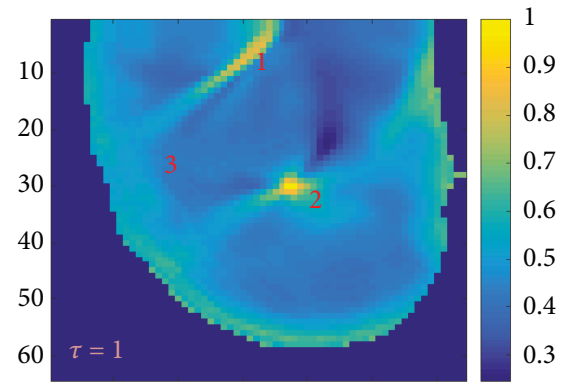

$\begin{array}{llllll}10 & 20 & 30 & 40 & 50 & 60\end{array}$

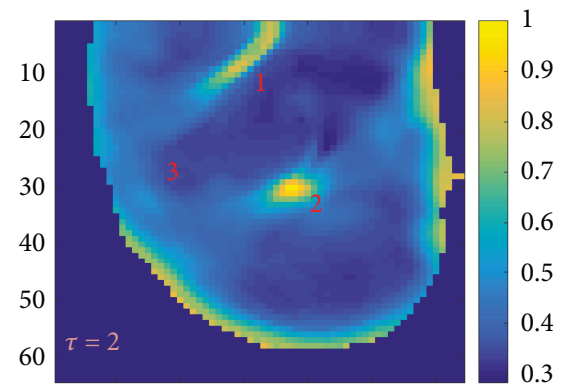

$\begin{array}{llllll}10 & 20 & 30 & 40 & 50 & 60\end{array}$

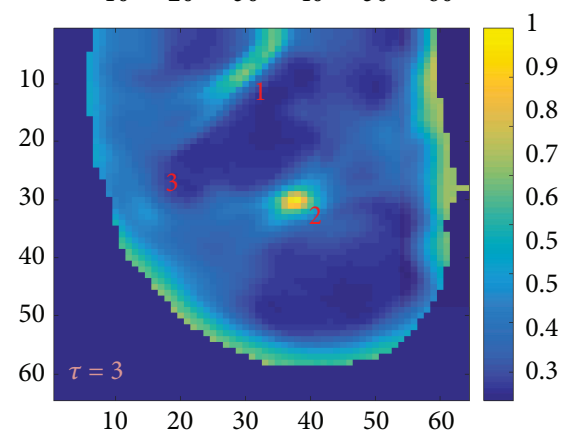

(b) 2D MSE maps

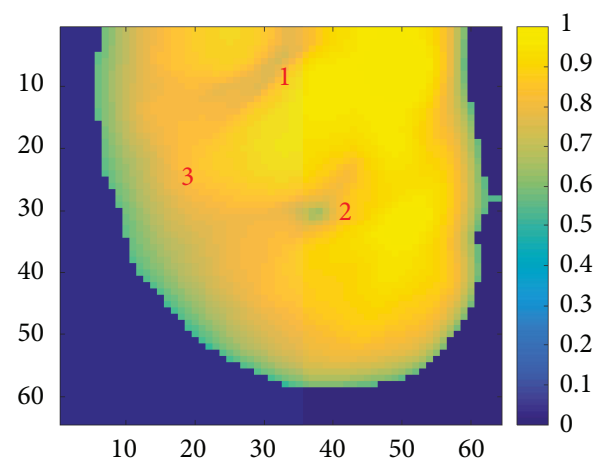

(c) 2D SE map

Figure 8: (a) Top panel shows a representative example of a figure-of- 8 reentry. Pixel locations "1" and " 2 " represent rotor core region and " 3 " represent rotor periphery; bottom panel shows corresponding voltage traces at those pixel locations. (b) Normalized 2D MSE maps; top panel with scale factor $\tau=1$; middle panel with $\tau=2$; bottom panel with $\tau=3$, correctly identifying rotor core regions; (c) normalized 2D SE map with lower SE values at the rotor core.

choice of scaling factor. The results from this study suggest that a scale factor of $\tau=3$ may be a reasonable starting point for many applications, but clinical validation is needed.

In addition, our analysis was limited to relatively small number of datasets. More rigorous evaluation using a larger number of datasets is critical in order to validate these findings for ECG discrimination as well as for rotor identification. Finally, we did not specifically evaluate ex vivo examples of AF but only of more organized cardiac arrhythmias to determine critical rotor elements. Given the higher-order 
complexity associated with AF, further study is needed in experimental models of AF to validate the use of MSE for characterization of rotors in these arrhythmia examples.

\section{Conclusions}

An improved MSE technique with nearest-neighbor moving-average kernel was developed to eliminate the systematic bias from one-sided averaging. The results demonstrate that MSE technique can be successfully used for the analysis of nonlinear and nonstationary short time series physiological data. Compared to the commonly used SE approach, MSE robustly estimated complexity with short time series data with various noises such as white, pink, and brown noises. The MSE discriminated NSR and AF on single-lead ECG of $10 \mathrm{~s}$ recordings without any preprocessing steps and precisely identified the pivot point of the rotors with $3 \mathrm{~s}$ optical mapping data from isolated rabbit hearts by providing better contrast between the rotor core and the periphery region when compared to the SE approach. Wide-range application of this technique on a variety of time series data can open new avenues for analysis and interpretation.

\section{Future Work}

Future work will focus on further validating the efficacy of NSR and AF discrimination on a larger dataset. Also, the MSE algorithm will be validated with a variety of rotor data for accurate identification of ablation targets using both optical mapping and intracardiac electrograms that can guide patient-specific mapping and ablation.

\section{Ethical Approval}

In this study no animal studies were performed. Data from previous animal studies were used for which all applicable international, national, and/or institutional guidelines for the care and use of animals were followed. This article does not contain any studies with human participants performed by any of the authors.

\section{Conflicts of Interest}

S. P. Arunachalam declares that he has no conflict of interest. S. Kapa declares that he has no conflict of interest. S. K. Mulpuru declares that he has no conflict of interest. P. A. Friedman declares that he has no conflict of interest. E. G. Tolkacheva declares that she has no conflict of interest.

\section{Acknowledgments}

This study was funded by the National Science Foundation Grants CAREER PHY-125541 and DCSD 1662250; and the National Institute of Health (NIH) Grant R21HL128790.

\section{Supplementary Materials}

Supplementary 1. SV1: phase movie of the single rotor from optical mapping of isolated rabbit heart.
Supplementary 2. SV2: phase movie of the figure-of-8 reentry from optical mapping of isolated rabbit heart.

\section{References}

[1] N. P. Reddy, "Book review: biomedical signal analysis: a case-study approach, by Rangaraja M. Rangayyan," Annals of Biomedical Engineering, vol. 30, no. 7, pp. 983-983, 2002.

[2] M. Kutz, Standard Handbook of Biomedical Engineering and Design, McGraw-Hill, New York, NY, USA, 2003.

[3] M. Baumert, A. Porta, and A. Cichocki, "Biomedical signal processing: from a conceptual framework to clinical applications [scanning the issue]," Proceedings of the IEEE, vol. 104, no. 2, pp. 220-222, 2016.

[4] P. F. Lin, M. T. Lo, J. Tsao, Y. C. Chang, C. Lin, and Y. L. Ho, "Correlations between the signal complexity of cerebral and cardiac electrical activity: a multiscale entropy analysis," PLoS One, vol. 9, no. 2, article e87798, 2014.

[5] C. E. Shannon, "A mathematical theory of communication," Bell System Technical Journal, vol. 27, no. 3, pp. 379-423, 1948.

[6] A. N. Kolmogorov, "Entropy per unit time as a metric invariant of automorphism," Academy of Sciences, vol. 124, pp. 754-755, 1959.

[7] G. E. Powell and I. C. Percival, "A spectral entropy method for distinguishing regular and irregular motion of Hamiltonian systems," Journal of Physics A: Mathematical and General, vol. 12, no. 11, pp. 2053-2071, 1979.

[8] S. J. Roberts, W. Penny, and I. Rezek, “Temporal and spatial complexity measures for electroencephalogram based braincomputer interfacing," Medical \& Biological Engineering \& Computing, vol. 37, no. 1, pp. 93-98, 1999.

[9] O. A. Rosso, S. Blanco, J. Yordanova et al., "Wavelet entropy: a new tool for analysis of short duration brain electrical signals," Journal of Neuroscience Methods, vol. 105, no. 1, pp. 65-75, 2001.

[10] C. Bandt and B. Pompe, "Permutation entropy: a natural complexity measure for time series," Physical Review Letters, vol. 88, no. 17, 2002.

[11] S. Pincus, "Approximate entropy (ApEn) as a complexity measure," Chaos, vol. 5, no. 1, pp. 110-117, 1995.

[12] J. S. Richman and J. R. Moorman, "Physiological time-series analysis using approximate entropy and sample entropy," American Journal of Physiology-Heart and Circulatory Physiology, vol. 278, no. 6, pp. H2039-H2049, 2000.

[13] H. C. Fogedby, "On the phase-space approach to complexity," Journal of Statistical Physics, vol. 69, no. 1-2, pp. 411425, 1992.

[14] M. Costa, A. L. Goldberger, and C. K. Peng, "Multiscale entropy analysis of complex physiologic time series," Physical Review Letters, vol. 89, no. 6, article 068102, 2002.

[15] S. D. Wu, C. W. Wu, K. Y. Lee, and S. G. Lin, "Modified multiscale entropy for short-term time series analysis," Physica A: Statistical Mechanics and its Applications, vol. 392, no. 23, pp. 5865-5873, 2013.

[16] A. Humeau-Heurtier, "The multiscale entropy algorithm and its variants: a review," Entropy, vol. 17, no. 5, pp. 31103123, 2015.

[17] J. Gao, J. Hu, F. Liu, and Y. Cao, "Multiscale entropy analysis of biological signals: a fundamental bi-scaling law," Frontiers in Computational Neuroscience, vol. 9, 2015. 
[18] H. Azami and J. Escudero, "Improved multiscale permutation entropy for biomedical signal analysis: interpretation and application to electroencephalogram recordings," Biomedical Signal Processing and Control, vol. 23, pp. 28-41, 2016.

[19] Y. Zhang, S. Wei, Y. Long, and C. Liu, "Performance analysis of multiscale entropy for the assessment of ECG signal quality," Journal of Electrical and Computer Engineering, vol. 2015, Article ID 563915, 9 pages, 2015.

[20] W. Y. Pan, M. C. Su, H. T. Wu, M. C. Lin, I. Tsai, and C. K. Sun, "Multiscale entropy analysis of heart rate variability for assessing the severity of sleep disordered breathing," Entropy, vol. 17, no. 1, pp. 231-243, 2015.

[21] C. C. Wang, C. D. Chang, and B. C. Jiang, "Pattern recognition of multiscale entropy curve for ECG signal analysis," Applied Mechanics and Materials, vol. 195-196, pp. 603-607, 2012.

[22] J. Wang, X. Ning, J. Li, Q. Ma, Y. Xu, and C. Bian, "Multiscale entropy analysis of electrocardiogram," Sheng wu yi xue gong cheng xue za zhi= Journal of biomedical engineering= Shengwu yixue gongchengxue zazhi, vol. 24, no. 5, pp. 978980, 2007.

[23] M. Costa, A. L. Goldberger, and C. K. Peng, "Multiscale entropy analysis of biological signals," Physical Review E, vol. 71, no. 2, article 021906, 2005.

[24] R. Parasnis, A. Pawar, and M. Manivannan, "Multiscale entropy and poincare plot-based analysis of pulse rate variability and heart rate variability of ICU patients," in 2015 International Conference on Intelligent Informatics and Biomedical Sciences (ICIIBMS), pp. 290-295, Okinawa, Japan, November 2015.

[25] H. C. Chiu, Y. H. Lin, M. T. Lo et al., "Complexity of cardiac signals for predicting changes in alpha-waves after stress in patients undergoing cardiac catheterization," Scientific Reports, vol. 5, no. 1, article 13315, 2015.

[26] G. D. Clifford, F. Azuaje, and P. Mcsharry, "ECG statistics, noise, artifacts, and missing data," Advanced Methods and Tools for ECG Data Analysis, vol. 6, p. 18, 2006.

[27] O. El B'charri, R. Latif, K. Elmansouri, A. Abenaou, and W. Jenkal, "ECG signal performance de-noising assessment based on threshold tuning of dual-tree wavelet transform," Biomedical Engineering Online, vol. 16, no. 1, p. 26, 2017.

[28] A. L. Goldberger, L. A. N. Amaral, L. Glass et al., "PhysioBank, PhysioToolkit, and PhysioNet: components of a new research resource for complex physiologic signals," Circulation, vol. 101, no. 23, pp. e215-e220, 2000.

[29] S. P. Arunachalam, S. K. Mulpuru, P. A. Friedman, and E. G. Tolkacheva, "Feasibility of visualizing higher regions of Shannon entropy in atrial fibrillation patients," in 2015 37th Annual International Conference of the IEEE Engineering in Medicine and Biology Society (EMBC), pp. 4499-4502, Milan, Italy, August 2015.

[30] A. Matiukas, A. M. Pertsov, P. Kothari, A. Cram, and E. G. Tolkacheva, "Optical mapping of electrical heterogeneities in the heart during global ischemia," in 2009 Annual International Conference of the IEEE Engineering in Medicine and Biology Society, vol. 1, pp. 6321-6324, Minneapolis, MN, USA, September 2009.

[31] S. S. Chugh, R. Havmoeller, K. Narayanan et al., "Worldwide epidemiology of atrial fibrillation: a global burden of disease 2010 study," Circulation, vol. 129, no. 8, pp. 837-847, 2014.

[32] D. D. McManus, J. Lee, O. Maitas et al., "A novel application for the detection of an irregular pulse using an iPhone $4 \mathrm{~S}$ in patients with atrial fibrillation," Heart Rhythm, vol. 10, no. 3, pp. 315-319, 2013.

[33] E. J. Benjamin, P. S. Chen, D. E. Bild et al., "Prevention of atrial fibrillation: report from a National Heart, Lung, and Blood Institute workshop," Circulation, vol. 119, no. 4, pp. 606618, 2009.

[34] M. J. Patil, B. K. Khadse, and S. R. Suralkar, "Discrimination between atrial fibrillation (AF) \& normal sinus rhythm (NSR) using linear parameters," Pratibha: International Journal of Science, Spirituality, Business and Technology (IJSSBT), vol. 1, p. 108, 2012.

[35] H. Calkins, K. H. Kuck, R. Cappato et al., "2012 HRS/EHRA/ ECAS expert consensus statement on catheter and surgical ablation of atrial fibrillation: recommendations for patient selection, procedural techniques, patient management and follow-up, definitions, endpoints, and research trial design," Heart Rhythm, vol. 9, no. 4, pp. 632-696.e21, 2012.

[36] R. Weerasooriya, P. Khairy, J. Litalien et al., "Catheter ablation for atrial fibrillation: are results maintained at 5 years of follow-up?," Journal of the American College of Cardiology, vol. 57, no. 2, pp. 160-166, 2011.

[37] C. Medi, P. B. Sparks, J. B. Morton et al., "Pulmonary vein antral isolation for paroxysmal atrial fibrillation: results from long-term follow-up," Journal of Cardiovascular Electrophysiology, vol. 22, no. 2, pp. 137-141, 2010.

[38] W. S. Tzou, F. E. Marchlinski, E. S. Zado et al., "Long-term outcome after successful catheter ablation of atrial fibrillation," Circulation. Arrhythmia and Electrophysiology, vol. 3, no. 3, pp. 237-242, 2010.

[39] C. Pappone, G. Vicedomini, G. Augello et al., "Radiofrequency catheter ablation and antiarrhythmic drug therapy: a prospective, randomized, 4-year follow-up trial: the APAF study," Circulation Arrhythmia and Electrophysiology, vol. 4, no. 6, pp. 808-814, 2011.

[40] S. M. Narayan and J. A. Zaman, "Mechanistically based mapping of human cardiac fibrillation," The Journal of Physiology, vol. 594, no. 9, pp. 2399-2415, 2016.

[41] T. F. Chao, H. M. Tsao, Y. J. Lin et al., "Clinical outcome of catheter ablation in patients with nonparoxysmal atrial fibrillation: results of 3-year follow-up," Circulation Arrhythmia and Electrophysiology, vol. 5, no. 3, pp. 514-520, 2012. 


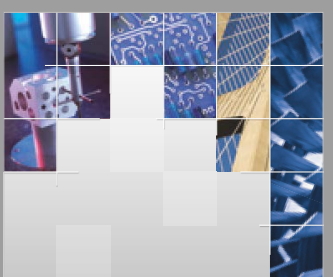

\section{Enfincering}
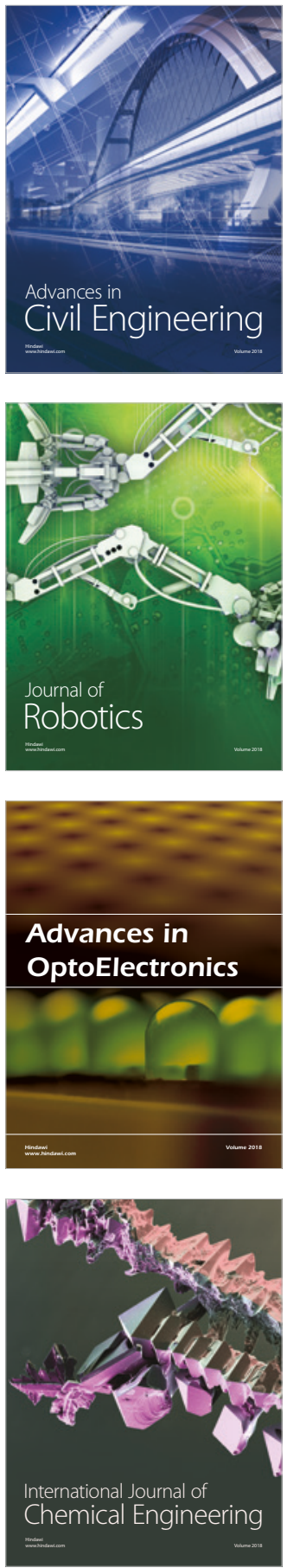

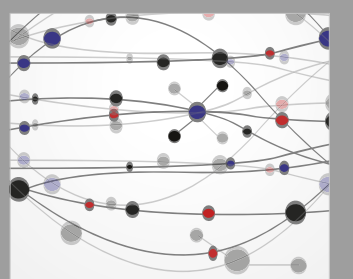

\section{Rotating \\ Machinery}

The Scientific World Journal

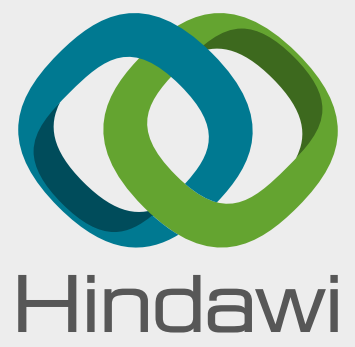

Submit your manuscripts at

www.hindawi.com
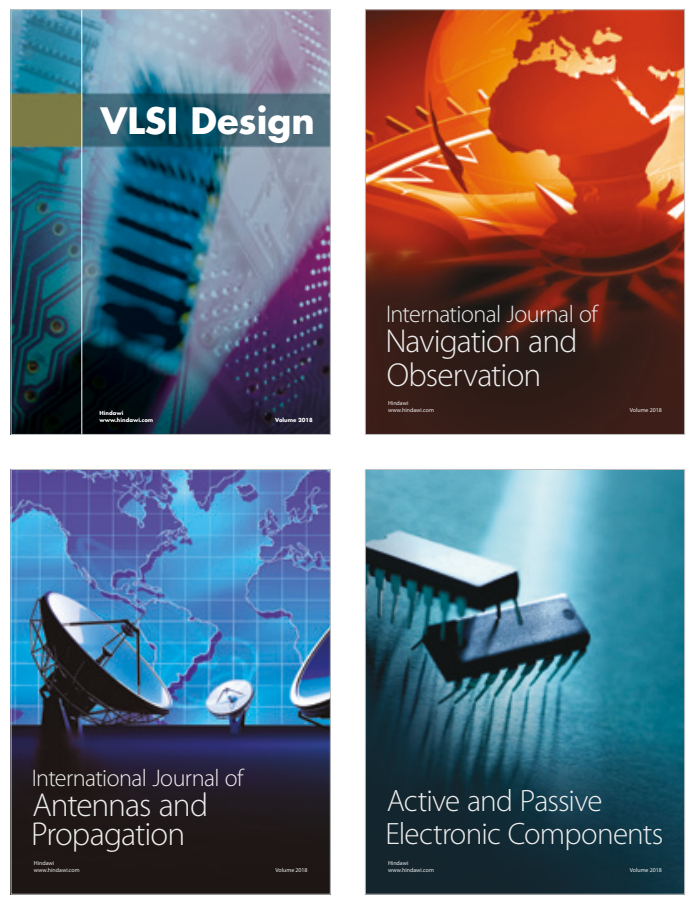
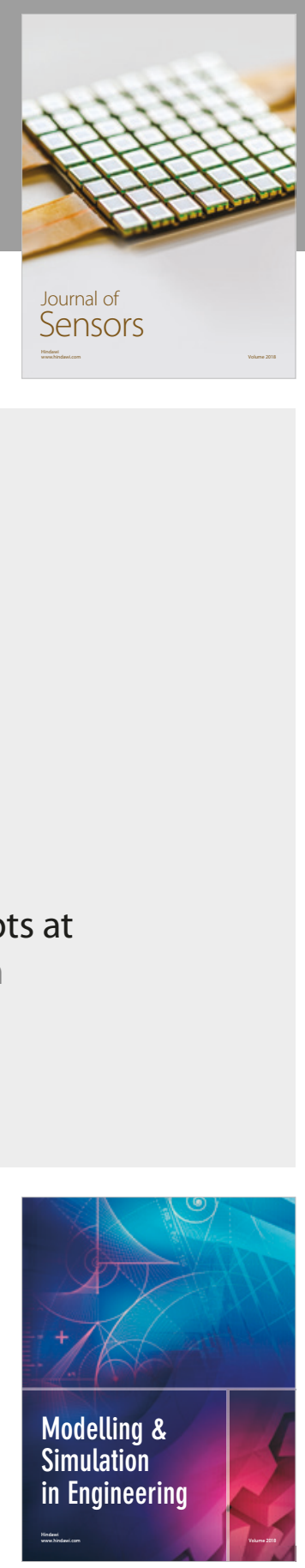

\section{Advances \\ Multimedia}
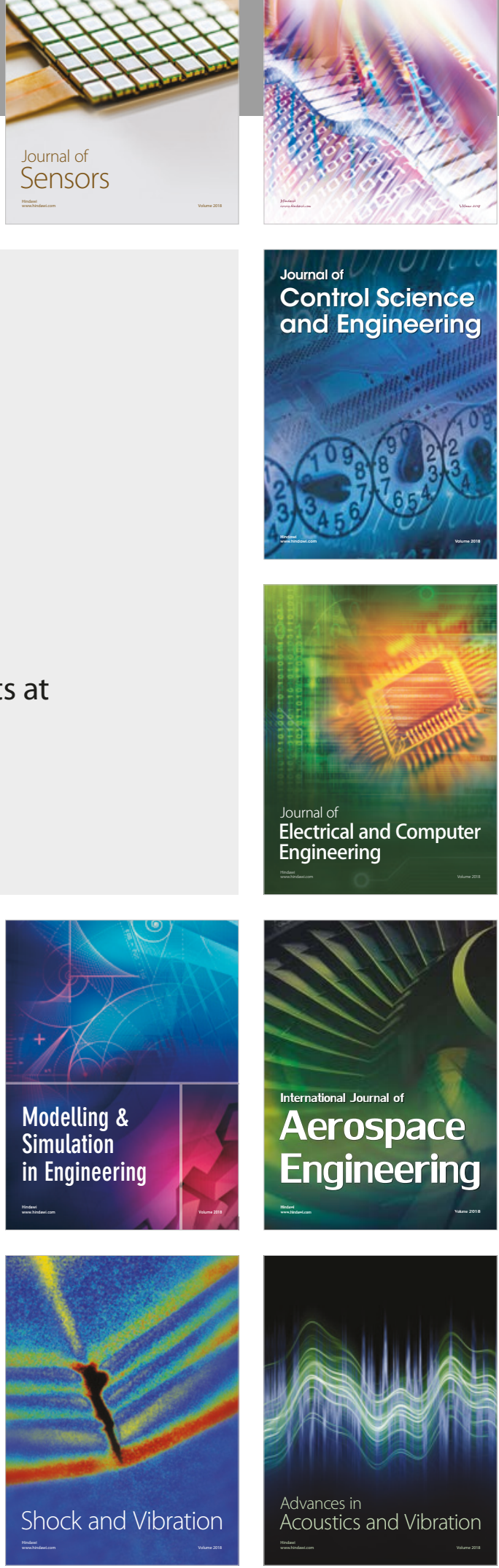